\title{
Real-space characterization of short-range order in Cu-Pd alloys
}

\author{
M. Rodewald \\ TH Darmstadt, Fachbereich Materialwissenschaft, Fachgebiet Strukturforschung, Petersenstrasse 23, D-64287 Darmstadt, Germany \\ K. Rodewald \\ Universität Hannover, Institut für Mineralogie, Welfengarten 1, D-30167 Hannover, Germany \\ P. De Meulenaere and G. Van Tendeloo \\ EMAT, Universiteit Antwerpen (RUCA), Groenenborgerlaan 171, B-2020 Antwerpen, Belgium
}

(Received 28 October 1996)

\begin{abstract}
$\mathrm{Cu}$-Pd alloys containing 10, 20, 30, 40, and 50 at. \% Pd and quenched from a temperature just above the ordering temperature $T_{c}$ are investigated by electron diffraction and high-resolution electron microscopy (HREM). The results show diffuse electron diffraction intensities at $\{100\}$ and $\{110\}$ positions for the alloy with 10 at. \% Pd, but with a characteristic twofold and fourfold splitting for the alloys with more than 10 at. \% Pd. High-resolution images show the formation of microdomains best developed between 20 and 30 at. \% Pd. A real-space characterization has been performed by applying videographic real-structure simulations revealing that the splitting of the diffuse maxima depends on the average distance between microdomains of $\mathrm{Cu}_{3} \mathrm{Au}$-type in antiphase with each other. By applying image processing routines on the HREM images, correlation vectors are identified which correspond to correlations between microdomains. [S0163-1829(97)11717-9]
\end{abstract}

\section{INTRODUCTION}

Copper-palladium alloys quenched from a temperature above $T_{c}$ show a characteristic diffraction pattern with diffuse intensities at or around the $\{100\}$ and $\{110\}$ positions due to short-range order (SRO). Cu-Pd alloys containing more than 13 at. \% Pd show twofold and fourfold splitting of these diffuse maxima. The separation of the split maxima increases with increasing Pd content. ${ }^{1-3}$

The relation between the local atomic arrangement above $T_{c}$ and shape and intensity of the split diffuse maxima has been the aim of many publications over the past forty years. One of the first papers on $\mathrm{SRO}$ in $\mathrm{Cu}_{3} \mathrm{Pd}$ was published by Watanabe $^{1}$ in 1959. The author interprets the diffuse intensity in the diffraction pattern by assuming that short chains of one-dimensional antiphase domains, with the same period of step shift as in the ordered state, may exist randomly in a disordered state. In 1973 a qualitative study of the diffuse intensities of $\mathrm{Cu}-\mathrm{Pd}$ alloys in the composition range between 13 and 60 at. \% Pd has been performed by electron diffraction. $^{2}$ The authors used the Fermi surface imaging theory proposed by Sato and Toth $^{4}$ for long-period structures. They concluded that the pair interaction potential in the $\mathrm{Cu}-\mathrm{Pd}$ system originates mainly from conduction electrons and that diffuse scattering reflects the flat section of the Fermi surface. In addition to this, Kubo and Adachi ${ }^{5}$ showed that the splitting can also be related to the Fermi wave vector $K_{F}$. In 1974 Hashimoto $^{6}$ proposed a correlative microdomain model for short-range ordered $\mathrm{Cu}_{3} \mathrm{Pd}$. He stated the existence of small ordered microdomains of $\mathrm{Cu}_{3} \mathrm{Au}$-type mutually antiphase correlated within a disordered matrix, which causes the fine structure of the diffuse scattering. Ohshima et al. ${ }^{7}$ quantitatively measured the diffuse x-ray-diffraction intensities for $\mathrm{Cu}-29.8$ at. \% Pd and determined the Warren-
Cowley SRO parameters. They pointed out that higher order parameters (beyond the 15th shell) play an important role in the appearance of the split diffuse maxima, although their magnitudes are small compared with those of lower order parameters. Gyorrfy and Stocks ${ }^{8}$ calculated the Fermi surfaces for disordered $\mathrm{Cu}-\mathrm{Pd}$ alloys and stated that the characteristic composition-dependent splitting of the diffuse maxima is due to parallel sheets of flat Fermi surface, and that the positions are directly related to the Fermi vector.

In 1983, Van Tendeloo and Amelinckx ${ }^{9}$ revealed, by high-resolution electron microscopy, evidence for the presence of microdomains in $\mathrm{Cu}_{70} \mathrm{Pd}_{30}$ alloys quenched from above $T_{c}$. Tanaka and Ohshima ${ }^{10}$ investigated alloys with 21 and 29.8 at. \% Pd, quenched from the SRO state, by highresolution electron microscopy. The authors suggested the existence of small ordered regions in a disordered matrix and proposed the existence of antiphase correlations, but showed no direct proof of this. A quantitative study of SRO diffuse scattering from the disordered state in the composition range from 8 to 42 at. $\% \mathrm{Pd}$ was performed by Saha et al. ${ }^{3}$ It showed that for $\mathrm{Cu}-\mathrm{Pd}$ alloys with $\mathrm{Pd}$ contents below 13 at. \% Pd no splitting of the diffuse intensity occurs. The structural interpretation of the diffuse intensities was performed based on the correlative microdomain model of Hashimoto. ${ }^{6}$ The existence of small ordered domains of $\mathrm{Cu}_{3} \mathrm{Au}$ type correlated with each other was assumed. With the aid of a simulation program, Suzuki et al. ${ }^{11}$ and Ohshima et $a l^{12}$ tried to generate possible local atomic arrangements with the size of $10 \times 10 \times 10 \mathrm{fcc}$ unit cells using the SRO parameters, but the results did not show splitting of the maxima. However, they were able to reveal, in good agreement with the result of Ohshima et al., ${ }^{7}$ that higher order parameters (beyond the 15th shell) play an important role in the appearance of the split diffuse maxima. 

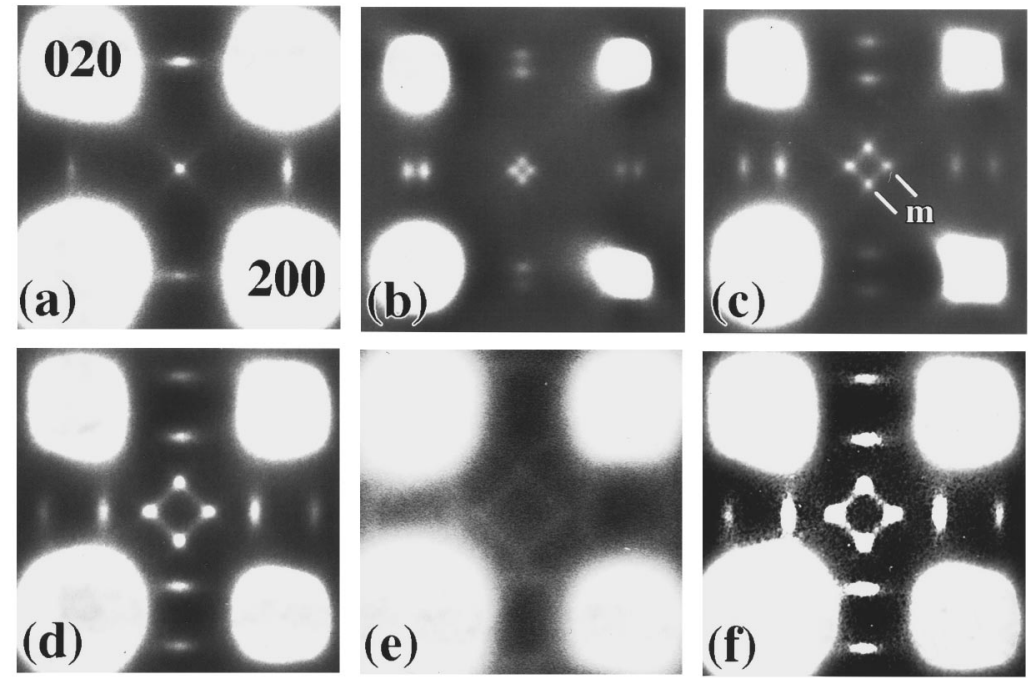

FIG. 1. Electron diffraction patterns of different $\mathrm{Cu}-\mathrm{Pd}$ alloys quenched from a temperature above $T_{c}$ ([001] incidence). (a) $\mathrm{Cu}_{90} \mathrm{Pd}_{10}$, (b) $\mathrm{Cu}_{80} \mathrm{Pd}_{20}$, (c) $\mathrm{Cu}_{70} \mathrm{Pd}_{30}$ (splitting-distance $m$ indicated), (d) $\mathrm{Cu}_{60} \mathrm{Pd}_{40}$, (e) $\mathrm{CuPd}$, (f) same as (d), but with overenhanced contrast to visualize the diffuse streaks.
In spite of these numerous studies on the binary $\mathrm{Cu}-\mathrm{Pd}$ alloy system, it is still questionable to which extent variations in the diffuse intensities of the diffraction pattern are related to the atomic arrangement in real space. In the present study, we use high-resolution electron microscopy (HREM) and electron diffraction to investigate the SRO state of $\mathrm{Cu}$-Pd alloys in the composition range between 10 and 50 at. \% Pd. The HREM images are evaluated applying recent image processing routines. ${ }^{13}$ In addition, the videographic simulation procedure ${ }^{14,15}$ is applied on the $\mathrm{Cu}-\mathrm{Pd}$ alloys. The comparison of the results obtained from the experimental images and from the videographic simulations is used to develop a structural interpretation for the characteristic shape of the diffuse maxima in the $\mathrm{Cu}-\mathrm{Pd}$ system.

\section{EXPERIMENT}

$\mathrm{Cu}-\mathrm{Pd}$ alloys containing 10, 20, 30, 40, and 50 at. \% Pd were prepared by melting the pure metals $\mathrm{Cu}$ and $\mathrm{Pd}$ mixed five times in the correct ratio in an arc-furnace under 0.5 bar argon atmosphere. The ingots were encapsulated in evacuated quartz tubes and annealed for homogenization at $1100{ }^{\circ} \mathrm{C}$ for one week. The composition of each alloy was checked by measuring the lattice constants using $\mathrm{x}$ rays and, independently from this, by chemical analysis. After homogenization the ingots were rolled into sheets with a thickness of about $0.1 \mathrm{~mm}$ and discs with a diameter of $3 \mathrm{~mm}$ were punched out of these sheets. The discs were again encapsulated in quartz-tubes and heated to $800{ }^{\circ} \mathrm{C}$ for $48 \mathrm{~h}$ to heal dislocations introduced by the rolling. Next the capsules were quenched in ice water. Foils for the transmission electron microscopy (TEM) study were then thinned by twin-jet polishing. For alloys with less than 25 at. \% $\mathrm{Pd}$ an electrolytic solution of 13.3 vol. $\% \mathrm{HNO}_{3}(65 \%)$ and 87.7 vol. \% methanol was used $(U=28 \mathrm{~V}, I \approx 1 \mathrm{~A})$ and, for alloys with more than 25 at. $\% \mathrm{Pd}$, a solution of 20 vol. $\% \mathrm{HNO}_{3}(65 \%)$ and 80 vol. $\%$ methanol $(U=33 \mathrm{~V}, I \approx 1.25 \mathrm{~A})$. The cleaning was performed in methanol.

High-resolution observations were made by using a 400 $\mathrm{kV}$ electron microscope (Jeol JEM 4000EX, $C s=1.07 \mathrm{~mm}$ ) equipped with a top-entry goniometer. A $200 \mathrm{kV}$ analytical electron microscope (Philips CM20) with EDX was used to perform chemical analysis. For image analysis the observed micrographs were digitalized with 256 grey levels from positive prints with the aid of a computer aided image processing system. To assist the interpretation of the atomic configuration for the short-range ordered state of the $\mathrm{Cu}-\mathrm{Pd}$ alloys, the videographic simulation procedure was used ${ }^{14,15}$ on an IBM RS6000 computer.

\section{RESULTS}

\section{A. Electron diffraction}

The characteristic electron diffraction patterns of different $\mathrm{Cu}-\mathrm{Pd}$ alloys are shown in Fig. 1. Diffuse scattering is visible at and around the $\{100\}$ and $\{110\}$ positions, in agreement with all previous observations. For the alloys with more than 10 at. \% Pd a twofold splitting at the $\{100\}$ positions and a fourfold splitting at the $\{110\}$ positions is clearly visible. The splitting distance $m$ [indicated in Fig. 1(c)] shows a monotonic increase with increasing $\mathrm{Pd}$ content (Table I and Fig. 9). Note that $m$ is internally calibrated and measured relative to the distance between the fundamental spots (000) and (200). The fine structure of the diffuse intensities at the $\{110\}$ positions consists of four weak streaks with a slight curvature [overenhanced in Fig. 1(f)]. The positions of the fourfold split maxima correspond to the intersections of these diffuse streaks. Though it is hard to compare intensities of different electron diffraction patterns, one can qualitatively conclude that the intensity of the split diffuse maxima increases from 10 over 20 to 30 at. \% Pd and

TABLE I. Values of the splitting distance $m$ (measured in terms of the distance between the 000 and 200 spots) determined by electron diffraction.

\begin{tabular}{cc}
\hline \hline Pd content (at. \%) & $m$ \\
\hline 10 & - \\
20 & 0.069 \\
30 & 0.123 \\
40 & 0.209 \\
50 & 0.282 \\
\hline
\end{tabular}


then decreases again. The diffuse intensity in the $\mathrm{Cu}_{50} \mathrm{Pd}_{50}$ alloy was much weaker than for alloys with 10 at. \% Pd. These observations are in good agreement with the results obtained in Refs. 2 and 3.

\section{B. Videographic simulations}

HREM images can only indirectly confirm a structural model by allowing to compare the experimental image contrast with the simulated image contrast of the proposed structure model. Because of the lack of suitable models for SRO, earlier TEM investigations ${ }^{9,10}$ could not be interpreted completely in terms of the real structure. In the present investigation the videographic method developed by $\operatorname{Rahman}^{14}$ and Rahman and Rodewald ${ }^{15}$ has been used for the generation of real-structure models for different $\mathrm{Cu}$-Pd alloys, and it has then to be proved that they are in agreement with the highresolution observations.

To simulate a real structure using the videographic method, a set of $n$ structure variants $\varphi_{j}(x, y, z) \quad(j$ $=1,2, \ldots, n)$ containing one or more atoms must be derived. These structure variants are distributed using a random variable $J$ taking the values $j(j=1, \ldots, n)$ with certain probabilities determined by the distribution function of $J$. In case of short-range ordering in an $A B$ alloy, the experimental shortrange order (SRO) parameters $\alpha_{l m n}$ can be used to derive influence factors $K_{l m n}$ for the correlation vectors $l m n$ connecting two atoms of type $A$ in the simulation $\left(m_{A}\right.$ and $m_{B}$ are the atomic fractions of $A$ and $B$ atoms in the alloy):

$$
K_{l m n}=\left[1-\left(1-\alpha_{l m n}\right) m_{B}\right] / m_{A}=\frac{P_{l m n}^{A A}}{m_{A}} .
$$

It therefore represents the probability $\left(P_{l m n}^{A A}\right)$ to find an $A-A$ pair connected with the interatomic vector $l m n$. During the simulation procedure the influence factors $K_{l m n}$ introduce deviations from a random distribution with the desired composition of the $A$ and $B$ atoms on the fcc sites. In this way a short-range-order model is generated. Based on the principles of the videographic simulation method, the structure variants $\varphi_{j}(x, y, z)$ (in this case single $\mathrm{Cu}$ or $\mathrm{Pd}$ atoms) are replaced by picture elements with different grey levels corresponding to the difference in scattering power. The resulting simulation can therefore be displayed and stored as a videographic image. Finally the real structure image $S(U, V, W)$, resulting from a three-dimensional simulation using correlation vectors, can be expressed as follows:

$$
S(U, V, W)=\sum_{u}^{U} \sum_{v}^{V} \sum_{w}^{W} \varphi_{J_{u v w}}(u v w),
$$

where $(u, v, w)=$ integers, $\varphi_{J u v w}(u v w)=$ structure variant of type $J$ at $u v w$ position, $J_{u v w}=$ random variable for $u v w$ position. To check the result of a simulation, the Fourier transform of the real-structure image $S(U, V, W)$ is calculated and compared with the experimental diffraction pattern.

The correlation vector simulations for the $\mathrm{Cu}-\mathrm{Pd}$ alloys were performed using the SRO parameters determined by Saha et al. ${ }^{3}$ It was necessary to use parameters up to the 33th shell in the simulation to obtain the correct splitting of the diffuse intensities. In the case of $\mathrm{Cu}_{3} \mathrm{Au}, \mathrm{Ni}_{4} \mathrm{Mo}$, and
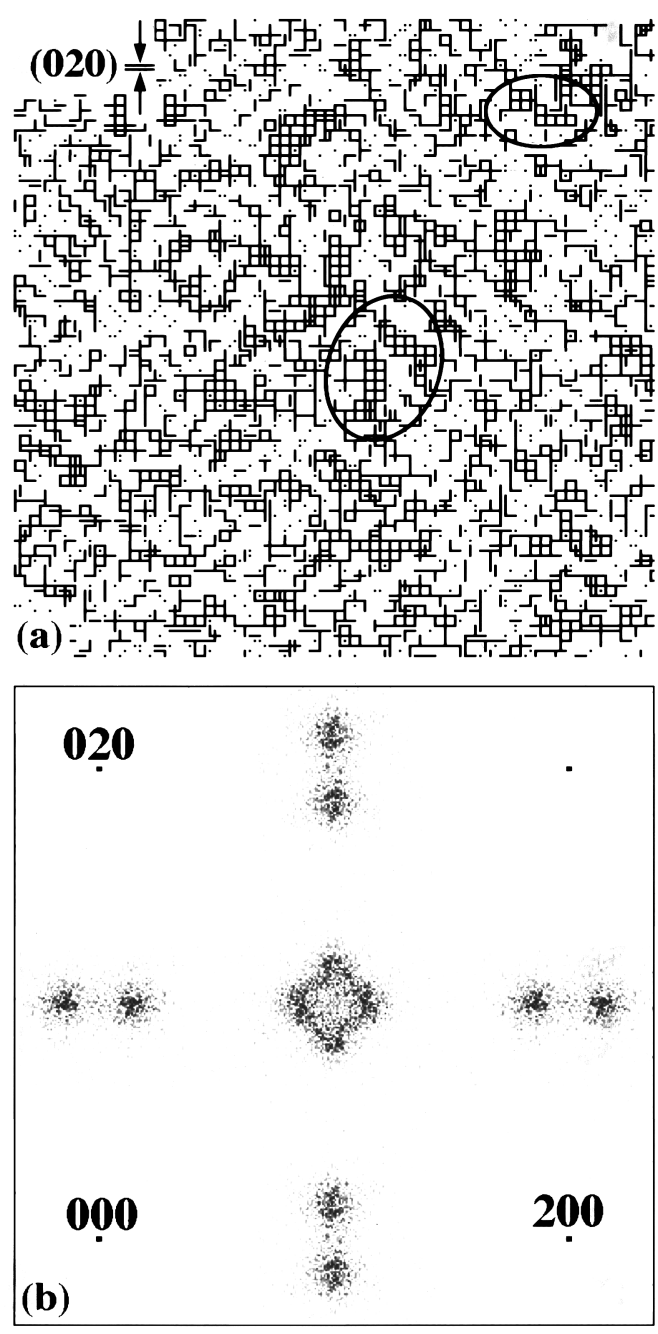

FIG. 2. (a) Two-dimensional section out of the threedimensional videographic real-structure simulation of $\mathrm{Cu}_{70} \mathrm{Pd}_{30}$ (lines have been added to visualize correlations of type $\langle 2,0\rangle$ ). The simulation was performed using SRO parameters determined by Saha et al. (1992). $L 1_{2}$ domains standing in antiphase are encircled. (b) FT of the whole simulation field.

$\mathrm{Au}_{4} \mathrm{Mn}$ it was sufficient to use correlation vectors up to the 6th shell to simulate the short-range order of these alloys. ${ }^{15}$ This observation confirms the suggestion of Ref. 7 that higher order parameters (beyond the 15th shell) play an important role in the appearance of the split diffuse maxima. The simulations used for a final structural interpretation of the diffuse diffraction intensities contained more than 2 million atoms $(128 \times 128 \times 32$ unit cells). Figure $2(a)$ shows a two-dimensional section out of the videographic correlation vector simulation of $\mathrm{Cu}_{70} \mathrm{Pd}_{30}$ in the SRO state, Fig. 2(b) shows the Fourier transform of the whole three-dimensional simulation field. The splitting of the diffuse intensities at the $\{100\}$ and $\{110\}$ positions is clearly visible, and the splitdistance $m$ is exactly the same as the one found in experimental diffraction patterns of an alloy of this composition. The structural evaluation of the simulation shows the formation of small domains of $L 1_{2}\left(\mathrm{Cu}_{3} \mathrm{Au}\right)$ type in antiphase. Some of these domains are encircled in Fig. 2(a). It must be noted that the Fourier transform of a two-dimensional section out of the same simulation exhibits only the fourfold 


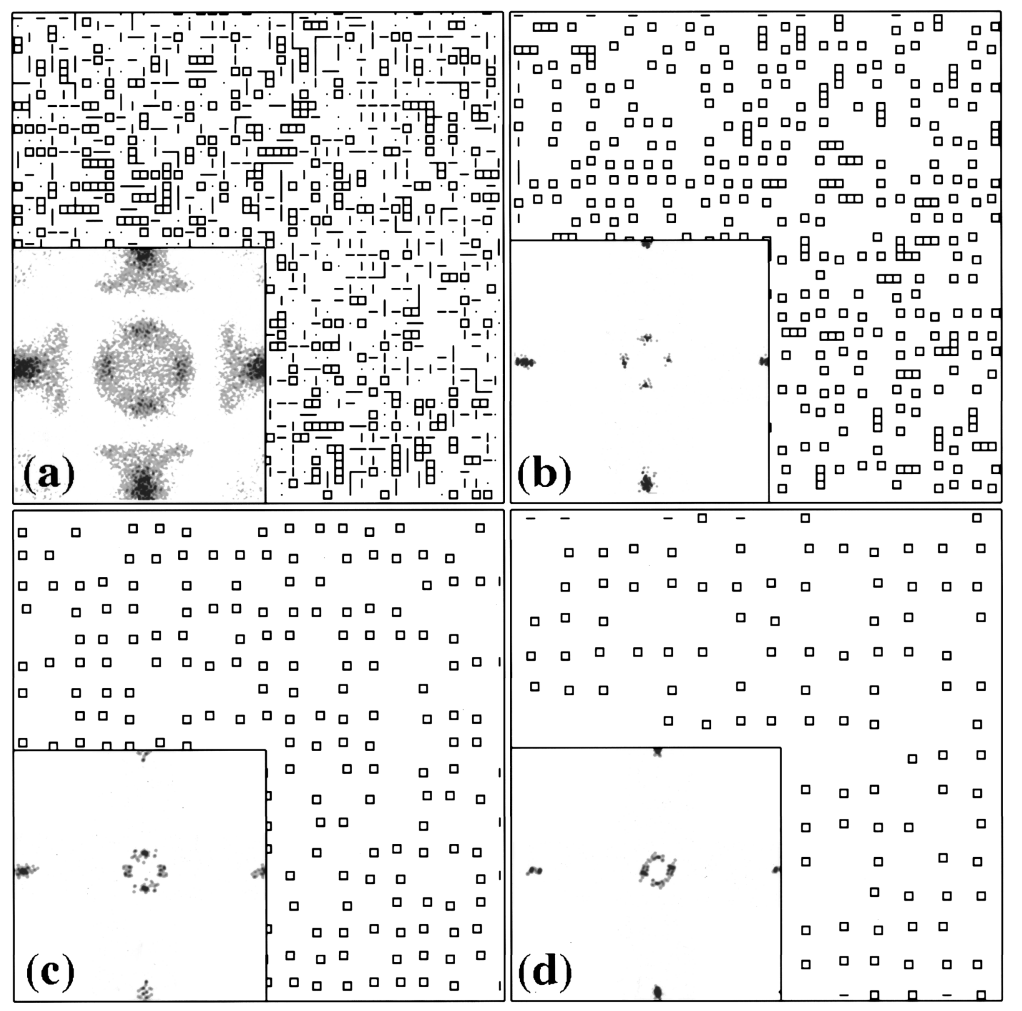

FIG. 3. Two-dimensional videographic simulation of a distribution of small $L 1_{2}$ domains with increasing antiphase distance in a disordered matrix (lines have been added to visualize correlations of type $\langle 2,0\rangle)$. The diffractograms are shown in the insets. (a) Antiphase-vector $\langle 2,1\rangle$ preferred, (b) antiphase-vector $\langle 4,1\rangle$ preferred, (c) antiphase-vector $\langle 6,1\rangle$ preferred, (d) antiphasevector $\langle 8,1\rangle$ preferred.

splitting and no splitting of the diffuse intensities at the $\{100\}$ positions due to the absence of antiphase correlations in the third dimension.

In order to prove this antiphase-domain model, twodimensional videographic simulations were performed distributing small $L 1_{2}$ domains (one unit cell) with a mean antiphase distance (Fig. 3). During these simulations it was revealed that the generation of an antiphase boundary perpendicular to another antiphase boundary at the same distance is not favorable. It results in a rotation over $45^{\circ}$ of the split diffuse maxima and was therefore suppressed in the simulation. This behavior shows similarities with results obtained by Rahman ${ }^{16}$ for $\mathrm{Cu}_{3} \mathrm{Au}$. Enlarging the ordered domains does not influence the splitting, only the integral intensity of the diffuse maxima increases with increasing domain size. The final comparison of simulations representing different compositions shows that the splitting of the diffuse maxima depends on the average distance between the domains in antiphase, rather than on the domain size. The most important correlations are therefore vectors of type $\langle n, 1\rangle(n=2,4,6, \ldots)$ which connect the antiphase domains in the different $\mathrm{Cu}-\mathrm{Pd}$ alloys. At the same time $\mathrm{Pd}-\mathrm{Pd}$ configurations connected by the vector $\langle 1,1\rangle$ or by vectors of type $\langle 1+n, 1+n\rangle(n=2,4,6, \ldots)$ are avoided by the system.

\section{High-resolution electron microscopy}

In order to prove or reject the models obtained from the videographic simulations, high-resolution electron microscopy images were taken for the different $\mathrm{Cu}-\mathrm{Pd}$ alloys. Figures 4(a) and 4(b) show bright field HREM images along the [001] zone of Cu-Pd alloys with 20 and 30 at. \% Pd, respectively. The fcc basic structure is clearly seen. Moreover, a random fragmentation in microdomains, containing approxi- mately 9 to 15 unit cells, is also visible. In this context, it should be noted that it was not possible under these imaging conditions to reveal microdomains in alloys with 10 at. \% or more than 40 at. $\% \mathrm{Pd}$.

The HREM images will now be interpreted in order to
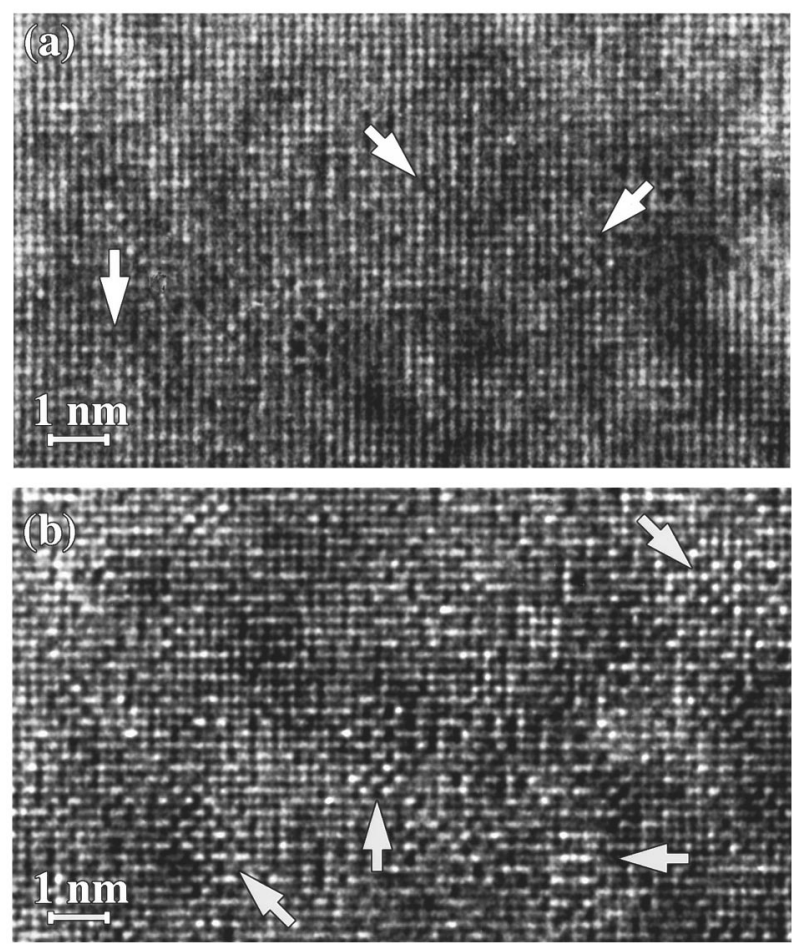

FIG. 4. HREM image of $\mathrm{Cu}_{80} \mathrm{Pd}_{20}$ (a) and $\mathrm{Cu}_{70} \mathrm{Pd}_{30}$ (b) quenched from above $T_{c}$ ([001] incidence). Some microdomains are indicated by arrows. 

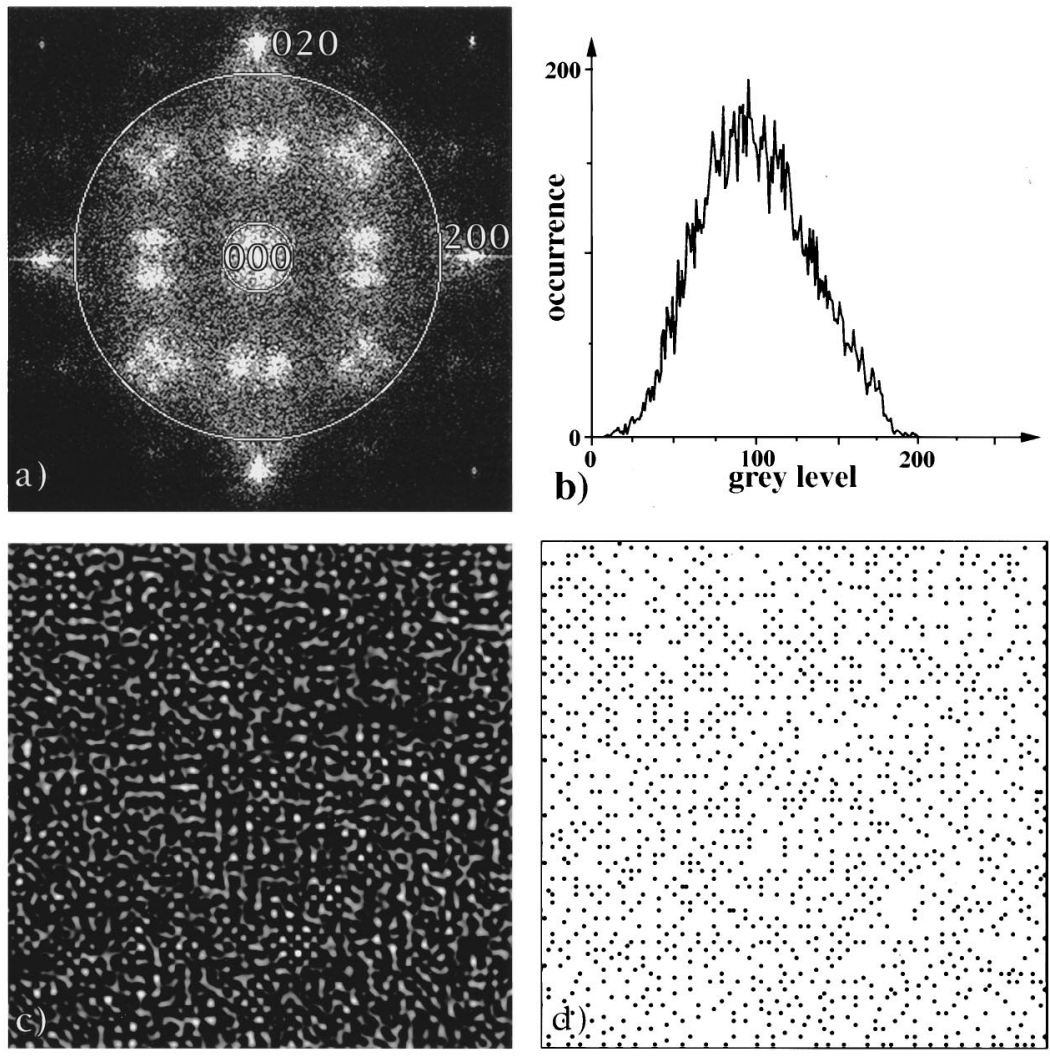

FIG. 5. Illustration of the image processing routine to convert the HREM image of Fig. 4(b) to a distribution of dots containing approximately the same SRO content. (a) Diffractogram of the original HREM image, (b) histogram of the column dot's maxima of the HREM image, (c) result after inverse Fourier transform of the diffuse intensity selected in (a) by the indicated ring filter, (d) distribution of dots, indicating the positions of the local maximum intensities in (c). For (c) and (d), only a part of the result is shown. find the most important correlations which induce the diffuse intensity splitting. It is first checked whether the SRO information of interest is indeed present in the corresponding HREM images. Figure 5(a) shows the diffractogram (i.e., the intensities of the calculated Fourier transform) calculated from Fig. 4(b) (30 at. \% Pd). Clearly, the intensities are much weaker and more spread out, as compared to the electron diffraction pattern. This is due to the small area which is taken into account, resulting in a limited statistics. Importantly, the splitting of the (110) and (100) reflections is still present, indicating that the corresponding SRO information can be retrieved from the HREM image. From this diffractogram, two-dimensional SRO parameters $\alpha_{l m}$ can be calculated by an inverse. Fourier transformation of the diffuse intensity:

$$
\alpha_{l m n}=\frac{1}{m_{A} m_{B} N V^{*}} \int_{V^{*}} I_{D}(\mathbf{g}) e^{-2 \pi \mathbf{g r}_{l m}} d \mathbf{g} .
$$

The values for the closest neighbors and for some vectors of the type $\langle n, 1\rangle$ are given in Table II. Caution has to be taken in the interpretation of these numbers: the values suffer from a poor statistics and from the dynamical scattering of electron diffraction. Moreover, only projected (two-dimensional) SRO parameters can be calculated from HREM. Nevertheless, they give very useful indications on the importance of some correlations. In Table II, Pd-Pd vectors of the type $\langle 4,1\rangle,\langle 6,1\rangle$, and $\langle 2,0\rangle$ occur relatively frequently. The very low value for $\alpha_{1,0}$ indicates that the system tries to avoid nearest neighbors of the same kind. The high value of $\alpha_{2,0}$ is clearly related to the presence of the projected $L 1_{2}$ and/or the $L 1_{0}$ clusters. The importance of the correlations for larger vectors is more difficult to assess, since these correlations are highly influenced by the correlations over shorter distances. As pointed out in the Introduction and in Sec.
III B, long-range correlations are needed to explain the splitting of the diffuse diffraction peaks. Because it is difficult to predict from the SRO parameters which parameter(s) precisely contribute(s) to this splitting, we use the real-space information present in the HREM image of Figs. 4(a) and 4(b). One can easily derive that the diffuse intensity

TABLE II. Two-dimensional SRO parameters, measured via the diffractogram of the HREM image of Fig. 4(b). The coordinates of the vectors are measured with respect to the smallest possible unit cell of the square [001]-projected fcc lattice. To convert them into fcc coordinates, they must be divided by two.

\begin{tabular}{cc}
\hline \hline,$m$ & $\alpha_{l m}$ \\
\hline 1,0 & -0.233 \\
1,1 & -0.065 \\
2,0 & +0.226 \\
2,1 & -0.079 \\
2,2 & +0.107 \\
3,0 & -0.134 \\
3,1 & -0.037 \\
3,2 & -0.050 \\
4,0 & +0.033 \\
4,1 & +0.061 \\
3,3 & -0.006 \\
5,1 & +0.020 \\
6,1 & +0.058 \\
7,1 & +0.012 \\
8,1 & +0.000 \\
9,1 & -0.005 \\
10,1 & -0.021 \\
\hline \hline
\end{tabular}



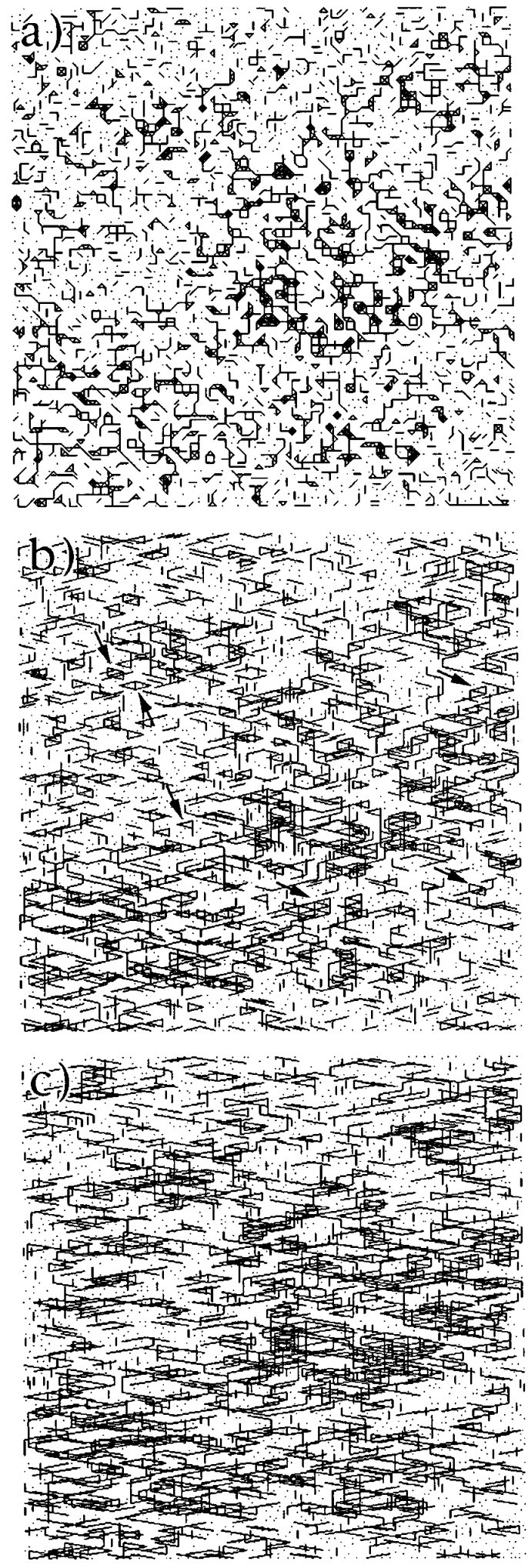

FIG. 6. Starting from Fig. 5(d), different pair correlations are visualized. (a) $\langle 1,1\rangle$ and $\langle 2,0\rangle$ vectors, (b) $[4,1],[4,-1]$, and $[0,2]$ vectors. Arrows indicate some typical clusters, (c) $[6,1],[6,-1]$, and $[0,2]$ vectors.

$I_{D}(g)$ in a diffractogram is expressed as (De Ridder et $a l .{ }^{17}$ ):

$$
I_{D}(\mathbf{g})=\sum_{j}\left\langle\left(f_{j}-\langle f\rangle\right)\left(f_{j+1}-\langle f\rangle\right)\right\rangle_{j} e^{\left(2 \pi i \mathbf{g} \cdot \mathbf{r}_{j}\right)}
$$
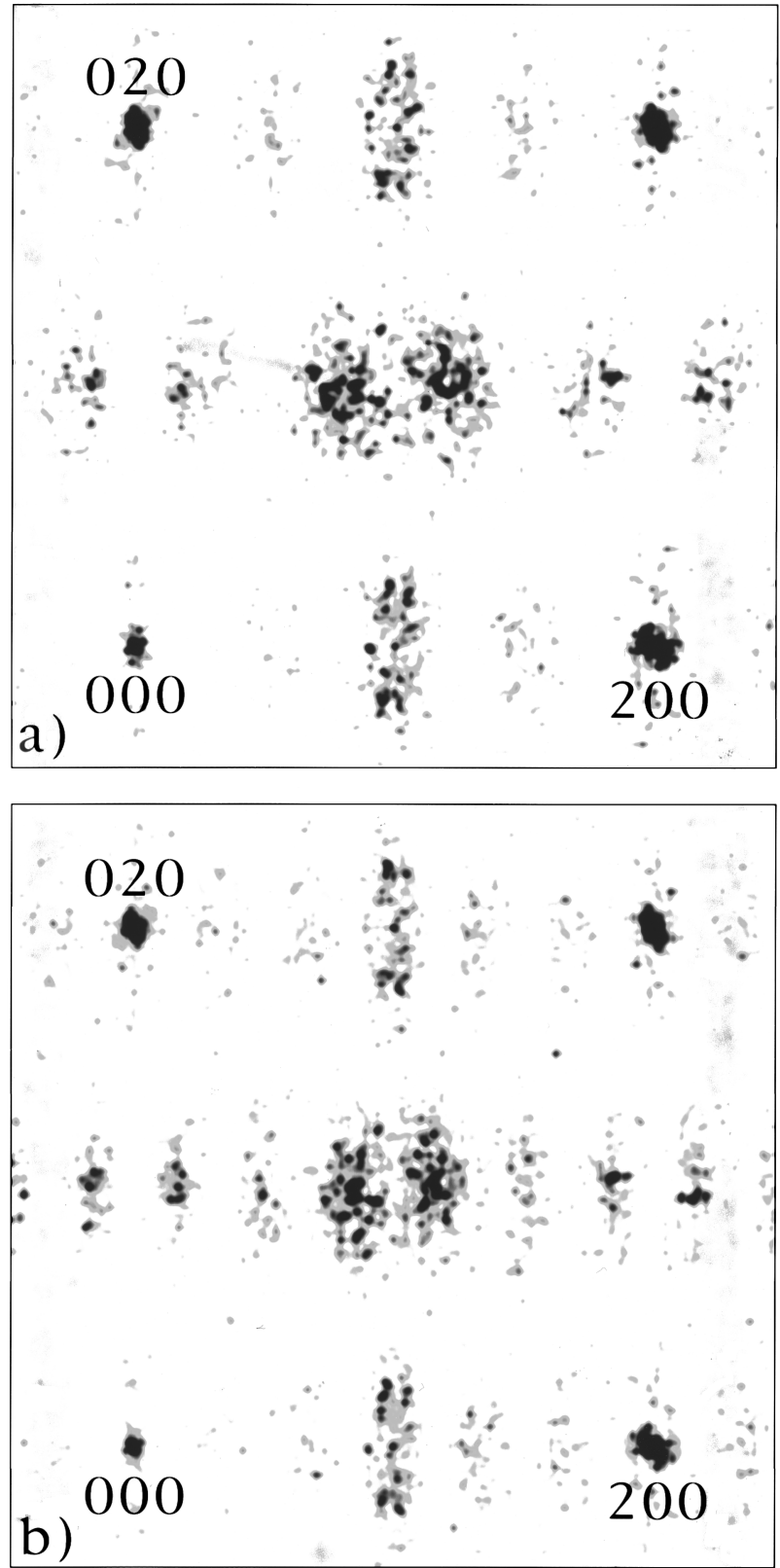

FIG. 7. Diffractograms for some subsets of points of Fig. 5(d). (a) and (b) result from the subset of points that can be connected by the vectors indicated in Fig. 6(b), respectively, 6(c).

This summation extends over all image points $r_{j}$ of the HREM image having different grey levels $f_{j}$ and an average grey level $\langle f\rangle$. It may be clear from this that very bright or very dark column dots may largely contribute to the diffuse intensity. Figure 5(b) illustrates the grey level distribution for Fig. 4(b), where only one characteristic pixel per column dot was taken into account (in this case the pixel with the maximum grey level). When compared to the average grey level, more bright column dots occur in this example than dark column dots. Therefore, we focus our attention to the intense columns only. It has also been shown that for such nonsymmetrical grey level distributions, the bright dots in the HREM image correspond to the atomic column positions in stead of to the empty channels in between the columns. ${ }^{13}$

To characterize the SRO, the experimental HREM image 


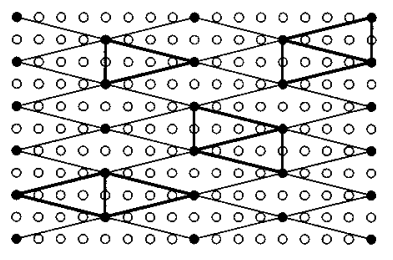

020

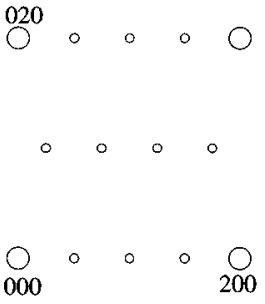

(a)

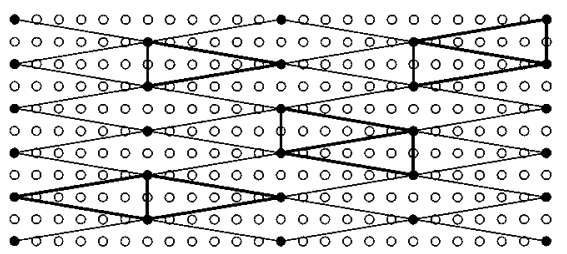

020

02000000

000000

$\bigcirc_{000} \circ \circ \circ \circ \circ \bigcirc$

(b)
FIG. 8. Hypothetical two-dimensional superstructures and corresponding diffraction patterns, containing the typical clusters observed in Fig. 6(b), respectively, 6(c). will now be converted to a binary black-and-white image which maintains the SRO information. This binary image is obtained by an image processing routine, which can be summarized as follows. After Fourier transformation of the original image, a ring filter is applied in Fourier space with a size indicated in Fig. 5(a): only the diffuse intensity of interest has been selected, while all Bragg peaks of the underlying fcc lattice are put to zero. Inverse Fourier transformation of the selected area yields Fig. 5(c). In this intermediate result, all local intensity maxima are sought and indicated in Fig. $5(\mathrm{~d})$. This is the binary image, indicating all column positions which give bright image intensities and which contribute to the SRO. It has been verified that Fig. 5(d) has qualitatively the same diffractogram as the original HREM image, including the spot splitting of interest. This preliminary result is much more manageable and correlations between columns can be found easier. More details on the image processing and the physical consequences of the method are described in Ref. 13.

Starting from Fig. 5(d), different correlations between selected columns are drawn in Fig. 6. Figure 6(a) shows all $\langle 1,1\rangle$ and $\langle 2,0\rangle$ correlations. One easily recognizes the $L 1_{2}$ and/or the $L 1_{0}$ microdomains. It must be noted that two overlapping $L 1_{2}$ microdomains in antiphase can result after projection in a two-dimensional $L 1_{0}$ configuration. Guided by the results of the videographic simulations, the correlations $\langle 4,1\rangle$ and $\langle 6,1\rangle$ are also studied. In Fig. 6(b), the combination of vectors $[4,1],[4,-1]$, and $[0,2]$ is shown. In some cases [see arrows in Fig. 6(b)], the vector [0,2] is connected with the head or tail of a $[4,1]$ or $[4,-1]$ vector, which sometimes results in typical clusters, such as the parallelograms or the isosceles triangles. As shown in Table II, these correlation vectors indeed occur frequently. It is also instructive to select from Fig. 5(d) only those points which are mutually correlated by one of the vectors $[4,1],[4,-1]$, or $[0,2]$. This subset of points represents $68 \%$ of the full set of points from Fig. 5(d). Its diffractogram is shown in Fig. 7(a). This diffractogram contains local intensity maxima at the positions $\left(\frac{1}{2} 00\right)$ and $\left(\frac{3}{2} 00\right)$ where there is no intensity in the original electron diffraction pattern. It is suggestive for a new twodimensional superstructure, as shown in Fig. 8(a). The typi- cal clusters in Fig. 6(b) can be retrieved from this superstructure [indicated by solid lines in Fig. 8(a)]. A similar reasoning has been applied for the $\langle 6,1\rangle$ family of vectors. Figure 6(c) shows the correlations $[6,1],[6,-1]$, and $[0,2]$. The corresponding subset of points results in the diffractogram of Fig. 7(b). The superstructure which generates the clusters of interest is shown in Fig. 8(b).

\section{DISCUSSION}

Irrespective of the absolute intensities of the diffuse scattering in the discussed $\mathrm{Cu}-\mathrm{Pd}$ alloys, the present system represents an almost complete transition from the (100) type of diffuse scattering $(m=0)$ to the $\left(1 \frac{1}{2} 0\right)$ type $[m=1 /(2 \sqrt{2})]$. The results from the simulations by the videographic method and their confirmation by HREM and subsequent image processing, show that $\mathrm{Pd}-\mathrm{Pd}$ connecting vectors of the type $\langle n, 1\rangle$ can explain the observed splitting. It will be discussed in a more general paper that the presence of antiphase boundaries between $L 1_{2}$ domains is related to the fact that the diffuse intensity always is positioned along the lines $\{x 10\}^{*}$. The average distance $n$ between two domains is then $1 / x$. $^{18}$

Below $T_{c}$ and depending on the composition, the $\mathrm{Cu}-\mathrm{Pd}$ alloy can reach either the $L 1_{2}$ structure, a one- or a twodimensional long-period antiphase boundary structure (LPAPB). These LPAPB's are characterized by a period $M$, whereby the splitting distance along one of the lines $\{x 10\}$ in the corresponding diffraction pattern is $1 / M$. The value $M$ then corresponds to the periodicity of the LPAPB structure. These phenomena have been studied in full detail in the past. ${ }^{4,19}$ Applying the same definition to the present observations of SRO, the relationship between $M$ and $m$ is $M=1 /(2 \sqrt{2} m)$. The temperature-dependent $M$ values from Broddin et $a l .{ }^{19}$ are converted to $m$ values and shown in the (concentration, $m$ ) diagram of Fig. 9. There is a clear correspondence between the $m$ values above and below $T_{c}$. Whereas below $T_{c}$, the $m$ is directly related to the periodicity of the structure, it is now found that above $T_{c}$ the corresponding $m$ indicates long-range correlations. Hence it can be stated that, in a first approximation, the tendency to form 


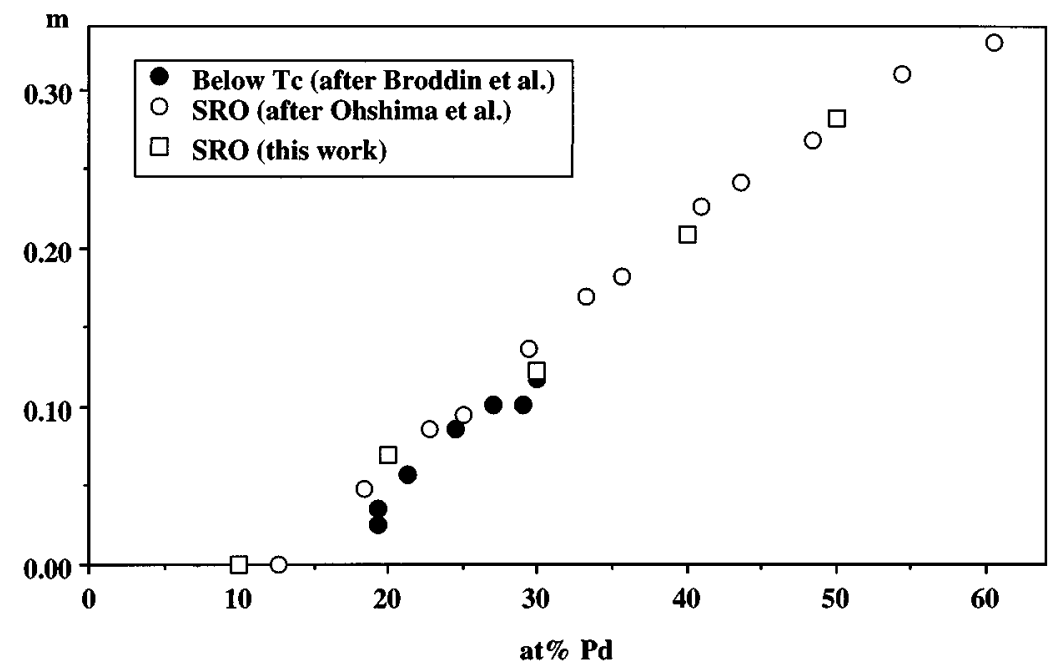

FIG. 9. (Concentration, $m$ ) diagram for observations both above and below $T_{c}$ (data from Ref. 2 and Ref. 19).

LPAPB's is already present in the SRO state of CuPd, even when the domain formation of the $L 1_{2}$ structure is not yet fully established.

The observation of the long-range correlations does not necessarily prove the existence of long-range interactions. But according to the Krivoglaz-Clapp-Moss formula, ${ }^{20,21,22}$ the maxima in the SRO diffuse intensity indicate the minima of the interatomic potential $V(g)$ in reciprocal space

$$
I(\mathbf{g})=C\left(1-\frac{T_{c} V(\mathbf{g})}{T V\left(\mathbf{g}_{m}\right)}\right)-1 .
$$

$T_{c}$ is the transition temperature, $g_{m}$ is the position where the potential $V$ reaches a minimum, and $C$ is a normalization constant. The occurrence of diffuse spot splitting hence indicates the existence of long-range interactions. The continuous increase of this splitting according to the Pd concentration can be explained by the changing number of electrons per atom ( $e / a$ ratio), as pointed out by Sato and Toth. ${ }^{4}$ In 1973, the theory of Sato and Toth has been applied to the $\mathrm{Cu}-\mathrm{Pd}$ system and the result is in good agreement with the experiment. $^{2}$

The direct-space observations in the present work are qualitative rather than quantitative. As for the videographic method, one should realize that real-space configurations derived from diffraction data by whatever algorithm, give only results for the pair correlations. All other cluster correlations, larger than pairs, cannot be retrieved from the diffraction pattern. In principle, HREM can also show larger clusters, since also the phase of the electron wave is of importance in the image formation. The correlations which are visualized by HREM are, however, correlations between atom columns, not between the atoms themselves. Due to the structure projection, the influence of defocus of the objective lens, and the unknown sample thickness, it is hard to retrieve the content of these columns. The image processing routine, described in the previous section, has the result that only those columns are selected which contribute to the SRO and which deviate from the average column composition, as shown by the histogram analysis [Fig. 5(b)]. Obviously, some important correlations have disappeared by this procedure, while other columns of less importance can be selected. The binary image showing the selected columns [as Fig. 5(d)] still con- tains much of the original SRO information, since its diffractogram clearly exhibits the diffuse spots on the correct positions. Of course, the original grey level HREM image contains still more SRO information, but the main advantage of this binary result is that pair correlations can easily be imaged. Moreover, a certain subset of points herein can be selected in order to identify specific characteristics of the original diffractogram.

\section{CONCLUSION}

In the past, research on the $\mathrm{SRO}$ in $\mathrm{Cu}-\mathrm{Pd}$ was focussed on reciprocal space observations. The present contribution reveals as much as possible the characteristics of SRO in real space. The results from the videographic simulations, starting from experimental SRO parameters, are confirmed by the projected configurations observed by HREM. Detailed analysis of the configurations, both from simulations and HREM observations, taught us that Pd-Pd correlation vectors of the type $\langle n, 1\rangle$ occur, where the inverse value of $n$ is proportional to the splitting distance of the diffuse diffraction spots. These characteristic vectors are shown to be related to the domain size in the LPAPB structures which occur below $T_{c}$. The corresponding Pd-Pd correlations can occur in the SRO state, independent of the occurrence of $L 1_{0}$ or $L 1_{2}$ clusters. The intensity of the diffuse spots reaches a maximum between 20 and 30 at. $\% \mathrm{Pd}$, when the $L 1_{2}$ domain size is largest. This is consistent with the fact that the perfect $L 1_{2}$ structure corresponds to an $A_{3} B$ composition. Diffuse streaking of the split reflections has been observed in the electron diffraction patterns. It is perpendicular to the split direction for the twofold splitting and along the $\langle 110\rangle$ directions for the fourfold splitting. The cause of this streaking will be discussed in a subsequent paper. $^{18}$

\section{ACKNOWLEDGMENT}

One of the authors (M.R.) acknowledges IUAP 48 for financial support during his stay at EMAT-RUCA. 
${ }^{1}$ D. Watanabe, J. Phys. Soc. Jpn. 14, 436 (1959).

${ }^{2}$ K. Ohshima and D. Watanabe, Acta Crystallogr. Sec. A 29, 520 (1973)

${ }^{3}$ D. K. Saha, K. Koga, and K. Ohshima, J. Phys. Condens. Matter 4, 10093 (1992).

${ }^{4}$ H. Sato and S. Toth, Phys. Rev. 124, 1833 (1961); 127, 469 (1962); J. Phys. Soc. Jpn. 17, Suppl. B II, 262 (1962b); in Alloying Behaviour of Concentrated Solid Solutions, edited by T. B. Massalski (Gordon, New York, 1965); Bull. Soc. Fr. Min. Cristallogr. 91, 557 (1968).

${ }^{5}$ S. Kubo and J. Adachi, J. Phys. Soc. Jpn. 35, 776 (1973).

${ }^{6}$ S. Hashimoto, Acta Crystallogr. Sec. A 30, 792 (1973).

${ }^{7}$ K. Ohshima, D. Watanabe, and J. Harada, Acta Crystallogr. Sec. A 32, 883 (1976).

${ }^{8}$ B. L. Gyorffy and G. M. Stocks, Phys. Rev. Lett. 50, 374 (1983).

${ }^{9}$ G. Van Tendeloo and S. Amelinckx, Phys. Status Solidi A 77, K9 (1983).

${ }^{10}$ N. Tanaka and K. Ohshima, Phys. Status Solidi A 81, 129 (1984).

${ }^{11}$ H. Suzuki, J. Harada, M. Matsui, and K. Adachi, Acta Crystallogr. Sec. A 38, 522 (1982).

${ }^{12}$ K. Ohshima, N. Iwao, and J. Harada, J. Phys. F 17, 1769 (1987).

${ }^{13}$ P. De Meulenaere, D. Van Dyck, G. Van Tendeloo, and J. Van Landuyt, Ultramicroscopy 60, 171 (1995); P. De Meulenaere, G.
Van Tendeloo, J. Van Landuyt, and D. Van Dyck, ibid. 60, 265 (1995).

${ }^{14}$ S. H. Rahman, Acta Crystallogr. Sec. A 49, 56 (1993).

${ }^{15}$ S. H. Rahman and M. Rodewald, Acta Crystallogr. Sec. A 51, 151 (1995).

${ }^{16}$ S. H. Rahman, Acta Crystallogr. Sec. A 49, 68 (1993b).

${ }^{17}$ R. De Ridder, G. Van Tendeloo, D. Van Dyck, and S. Amelinckx, Phys. Status Solidi A 38, 663 (1976); R. De Ridder, D. Van Dyck, G. Van Tendeloo, and S. Amelinckx, ibid. 40, 669 (1977); D. Van Dyck, R. De Ridder, G. Van Tendeloo, and S. Amelinckx, ibid. 43, 541 (1977).

${ }^{18}$ P. De Meulenaere, M. Rodewald, and G. Van Tendeloo (unpublished).

${ }^{19}$ D. Broddin, G. Van Tendeloo, J. Van Landuyt, S. Amelinckx, R. Portier, M. Guymont, and A. Loiseau, Philos. Mag. A 54, 395 (1986).

${ }^{20}$ M. Krivoglaz and A. Smirnov, The Theory of Order-Disorder in Alloys (McDonald, London, 1964).

${ }^{21}$ P. C. Clapp and S. C. Moss, Phys. Rev. 142, 418 (1966); 171, 754 (1968); S. C. Moss and P. C. Clapp, ibid. 171, 764 (1968).

${ }^{22}$ M. Krivoglaz, Theory of X-ray and Thermal Neutron Scattering by Real Crystals (Plenum, New York, 1969). 